\title{
Focus on In Silico Aptamer Synthesis New Food for Thought
}

\author{
E Alfinito*1 and R Cataldo ${ }^{2}$ \\ ${ }^{1}$ Department of Innovation Engineering, Italy \\ ${ }^{2}$ Department of Mathematics and Physics, Italy
}

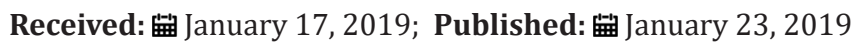

*Corresponding author: E Alfinito, Department of Innovation Engineering, Italy

\begin{abstract}
The use of aptamers (short single strands of DNA or RNA) in therapy, diagnosis and early detection of illness is a challenging field of research. Aptamers are artificially produced, tailored to best fit a specific target, and are a competitive alternative to antibodies. The in vivo procedures to synthetize aptamers are in continuous development, enlarging the scenario of the possible targets and improving the effectiveness of the products. However, as of today, these methods are still quite time consuming and expensive to allow mass production. On the other side, guessing the 3D structure of macromolecules is the task of several computational procedures, which have, over time, refined their predictive power and that can be used to look for the complexes with the highest stability. In the near future, hopefully, the joint use of in vitro and in silico techniques will accelerate and ameliorate the selection procedure. At the moment nor the in vitro neither the insilico procedures are flawless.
\end{abstract}

\section{Introduction}

The improved knowledge about the etiology of several diseases as well as the discovery of the epidemiologic aspects in the onset of some of them, is changing the approach to cure and prevention. Each disease is seen as a unique phenomenon, to be addressed looking at the personal history and genetic profile of the patient. As a consequence, therapy and prevention are becoming increasingly customized, and cures tend to be minimally invasive. On the other side, effectiveness has to be preserved, and to this aim, research mimics Nature. For example, immunotherapy, aims to contrast cancer by inducing an immune response against upregulated cells. In this regard, the design and production of new antibodies and aptamers for targeted therapies is the focus of many researches. Several monoclonal antibodies have been approved [1], while other are under clinical trials, despite the occurrence of quite severe side effects, like auto-inflammatory immune response, that can reduce the effectiveness of the treatment. Aptamers, like monoclonal antibodies, are synthetized to perfectly fit a specific target. Their selectivity, low size, short half-life, low immunogenicity, high specificity, and low production costs make them formidable competitors of antibodies in both diagnosis and therapy [2].

The production of aptamers started in 1990 [3], by means of the innovative SELEX technique, which is still in use both in the original version and also in several other updates [4]. It uses wide libraries of sequences to select, after several steps of severe selections, those with the highest affinity for the selected targets. With a conceptually similar procedure, ad hoc developed computer codes guess the possible shapes of several aptamer sequences and identify those with the highest affinity for the selected target [5]. The structure selection develops a multistep procedure that creates a pool of possible initial sequences, then samples the conformational space, producing the 3D shapes which are expected to be the most biologically relevant. Docking software select the best binding between a pair of macromolecules, using rigid or flexible roto-translations. The results are ranked according to the interaction energies between the two molecules, the desolvation and solvation energies associated with the interacting molecules, the entropic factors that occur upon binding [6].

\section{A Case Study: Aptamer-Angiopoietin Complexes}

In cancer therapy the role of angiopoietins in early detection is well established. As a matter of facts, both angiopoietin-1 (Ang1) and angiopoietin-2 (Ang2), and their natural receptors, Tie1, Tie2 appear as overexpressed in the presence of specific tumors [7] or blood diseases [8]. Angiopoietin-specific aptamers were selected both in vivo and in silico [9], although the in-silico predictions did not completely match with experimental data. In a recent paper a numerical investigation was used to predict the structure of 5 different aptamers [10] when complexed with Ang2. One of these sequences is Ang1-specific and used as a benchmark. 
The configurations of the complexes were obtained by using free software and ranked on the basis of an indicator which considers both the configurational and also the binding energy. This indicator was called effective affinity (EA). The procedure proposes hundreds of configurations for each sequence, whose mean properties were analyzed by using a complex network approach called Proteotronics $[11,12]$. In a nutshell, each complex was mapped into an impedance network whose topological and electrical features were used to produce an alternate ranking based on a novel indicator, Möbius (M) [13]. The mean features of each aptamer were in reasonable agreement with experimental data.9 However, after a deeper investigation, it clearly appeared that the samples given by the numerical procedure were not homogeneous. In particular, we found that the set of configurations produced for each aptamer was divided into two different types of conformers. In the conformer called hair, the aptamer is located on the head of the protein, in the other, called belt, the aptamer embraces the protein. Hair conformer reproduces the natural Ang2-Tie2 binding, while belt conformer suggests a new kind of binding. Both these conformers may result in high values of the effective affinity, because both correspond to complexes in which the aptamer and the target are very close to each other. In order to investigate the significance of the belt conformer, both EA and $\mathrm{M}$ indicators were used to perform a different scoring of the sequences [13]. The aptamer which is, in vivo, the best performing showed a prevalence of hair conformers among the highest score configurations, while a prevalence of belt conformers was detected among the highest score configurations of the aptamer which is, in vivo, the worst performing. The aptamers which are mutant sequences have not a specific characterization in terms of hair or belt conformers.

These results suggest different possible interpretations. For example, both the conformers can appear in real samples, although with different dissociation constants. In such a way, the relative abundance of hair/belt conformers in the pool of sequences representing each aptamer can be considered a measure of the complex stability. On the other hand, it has to be highlighted that the docking was performed by using the available Ang-2 crystallographic data, which refer, at the moment, to only a fragment of the protein. The protein works in vivo as a dimer, therefore a part of the surface which in simulations is assumed to be available for binding could, actually, be occupied by the other monomer. This makes the comparison between numerical simulations and experimental data quite questionable.

\section{Conclusion}

In silico docking is a powerful technique for the selection of aptamers with high affinity for an assigned target. Computational methods are becoming more and more rapid and effective, although a reliable ranking of their products, often several tens, still remains a challenging problem. In a recent investigation, we have shown that the introduction of a novel kind of scoring is necessary, especially when the software is unable to discriminate between quite different types of conformers, say, belt and hair. The present research opens the door to different conclusions. On one hand, both the conformers can be found in vivo, with different half-lives; the relative abundancies of the two conformers determines the stability of a real sample. On the other hand, it has to be emphasized that some of the belt conformers could not be realistic structures, since the aptamer occupies a part of protein surface which is not really available. Finally, an insilico investigation can be used, at present, only as a guide and an initial screening for the selection of high affinity aptamers, while crystallographic investigation still remains mandatory. To progress, it is necessary to gain more complete information about the target, so as design new indicators useful to ascertain the quality of the results.

\section{References}

1. Darvin P, Toor SM, Nair VS, Elkord E (2018) Immune checkpoint inhibitors: recent progress and potential biomarkers. Exp Mol Med 50(12): 165.

2. Pastor F (2016) Immune-checkpoint blockade aptamers as a feasible clinical alternative to monoclonal antibodies. Cancer Cell Microenviron 3(2): 1247.

3. Tuerk C, Gold L (1990) Systematic evolution of ligands by exponential enrichment: RNA ligands to bacteriophage T4 DNA polymerase. Science 249(4968): 505-510.

4. Cataldo R, Leuzzi M, Alfinito E (2018) Modelling and Development of Electrical Aptasensors: A Short Review. Chemosensors 6(2): 20.

5. Chushak Y, Stone MO (2009) In silico selection of RNA aptamers. Nucleic Acids Res 37(12): 87-87.

6. Du X, Li Y, Xia YL, Ai SM, Liang J, et al. (2016) Insights into protein-ligand interactions: mechanisms models and methods. Int J Mol Sci 17(2): 144.

7. Buehler D, Rush P, Hasenstein J R, Rice SR, Hafez GR, et al. (2013) Expression of angiopoietin-TIE system components in angiosarcoma. Mod Pathol 26(8): 1032.

8. Barber BE, Grigg MJ, Piera KA, William T, Cooper DJ, et al. (2018) Intravascular haemolysis in severe Plasmodium knowlesi malaria: association with endothelial activation microvascular dysfunction and acute kidney injury. Emerg Microb Infect 7(1): 106.

9. Hu WP, Kumar JV, Huang CJ, Chen WY (2015) Computational selection of RNA aptamer against angiopoietin-2 and experimental evaluation. BioMed Res Int.

10. Cataldo R, Ciriaco F, Alfinito E (2018) A validation strategy for in silico generated aptamers. Comput. Biol Chem 77: 123-130.

11. Alfinito E, Reggiani L, Cataldo R, De Nunzio G, Giotta L, et al. (2017) Modeling the microscopic electrical properties of thrombin binding aptamer (TBA) for label-free biosensors. Nanotechnology 28(6): 065502 .

12. Alfinito E, Reggiani L, Pousset J (2015) Proteotronics Electronic devices based on proteins. In Sensors: pp. 3-7.

13. Cataldo R, G Giotta L, Guascito MR, Alfinito E (2019) Assessing the Quality of in Silico Produced Biomolecules: The Discovery of a New Conformer J Phys Chem B in press. 
ISSN: 2574-1241

DOI: 10.26717/BJSTR.2019.13.002407

E Alfinito. Biomed J Sci \& Tech Res

(C) (i) This work is licensed under Creative

Submission Link: https://biomedres.us/submit-manuscript.php

$\begin{array}{ll}\text { BIOMEDICAL } & \text { Assets of Publishing with us } \\ \text { RESEARCHES } & \text { Global archiving of articles } \\ \text { - Immediate, unrestricted online access } \\ \text { https://biomedres.us/ }\end{array}$

